Pacific Journal of Mathematic 


\section{BOUNDARY BEHAVIOR OF RANDOM VALUED HEAT POLYNOMIAL EXPANSIONS}

\section{ROBERT B. Hughes}

This paper is concerned with random series of the form $\sum_{n=0}^{\infty} X_{n}(\omega) a_{n} v_{n}(x, t)$ where the $X_{n}$ 's are random variables, the $a_{n}$ 's are real numbers, and the $v_{n}$ 's are heat polynomials as introduced by P. C. Rosenbloom and D. V. Widder. The sequences $\left\{a_{n}\right\}$ are assumed to satisfy $\lim \sup _{n \rightarrow \infty}\left|a_{n}\right|^{2 / n}(2 n / e)=$ 1 which implies $\sum_{n=0}^{\infty} a_{n} v_{n}(x, t)$ has $|t|<1$ as its strip of convergence, i.e., it converges to a $C^{2}$-solution of the heat equation in $|t|<1$ and does not converge everywhere in any larger open strip. Associated with each sequence $\left\{\alpha_{n}\right\}$ is its classification number from $[0,1]$ which indicates how rapidly $a_{n}$ tends to zero. Assumptions are placed on the random variables which imply that for almost every $\omega$ the series $\sum_{n=0}^{\infty} X_{n}(\omega) a_{n} v_{n}(x, t)$ has $|t|<1$ as its strip of convergence.

The main results of the paper are two theorems. The first states that if $\left\{a_{n}\right\}$ has its classification number in $[0,1 / 2)$, then for almost every $\omega$ the lines $t=1$ and $t=-1$ form the natural boundary for $\sum_{n=0}^{\infty} X_{n}(\omega) a_{n} v_{n}(x, t)$. The second is concerned with sequences having their classification numbers in (1/2.1]. The conclusion implies that for almost every $\omega$ no interval of either of the lines $t=1$ or $t=-1$ is part of the natural boundary for $\sum_{n=0}^{\infty} X_{n}(\omega) a_{n} v_{n}(x, t)$.

The present work had it original motivation in the study of the boundary behavior of random power series. These are series of the form $\sum_{n=0}^{\infty} a_{n}(\omega) z^{n}$ where the $a_{n}$ 's are complex valued random variables and $z$ is a complex number. Reference [1] contains a history of results in this area. One of the early results which helped to motivate the first part of the proof of our Theorem 1 is due to Paley and Zygmund in a 1932 paper [see 6, p. 220]. In this theorem it is assumed that $\sum_{n=0}^{\infty} a_{n} z^{n}$ is an ordinary power series with a finite radius of convergence. Letting $\left\{\dot{\phi}_{n}\right\}$ be the sequence of Rademacher functions, the conclusion is that for almost every $\omega$ in $[0,1]$ the series $\sum_{n=0}^{\infty} \phi_{n}(\omega) a_{n} z^{n}$ has its circle of convergence as its natural boundary.

More recently [see 3] V. L. Shapiro has considered series of the form $\sum_{n=0}^{\infty} X_{n}(\omega) H_{n}(x)$ where the $X_{n}$ 's are random variables and

$$
\sum_{n=0}^{\infty} H_{n}(x)
$$

is the spherical harmonic representation of a harmonic function in the unit ball. The harmonic continuability across the boundary of the unit ball of the functions $\sum_{n=0}^{\infty} X_{n}(\omega) H_{n}(x)$ was investigated. This 
work further motivated the first part of the proof of our Theorem 1 and influenced our choice of the class of random variables to be considered.

2. Definitions and preliminary comments. For a point $\left(x_{0}, t_{0}\right)$ in the plane and a number $\rho>0$ we let

$$
S\left(x_{0}, t_{0} ; \rho\right)=\left\{(x, t):\left|x-x_{0}\right|<\rho \text { and }\left|t-t_{0}\right|<\rho\right\} \text {. }
$$

If $u(x, t)$ is a $C^{2}$-solution to the heat equation in the strip $|t|<\sigma$ we say the line $t=-\sigma(t=\sigma)$ is part of the natural boundary for $u$ in case for every $x_{0}$ and every $\rho>0$ there is no $C^{2}$-solution $v(x, t)$ in $S\left(x_{0},-\sigma ; \rho\right)\left(S\left(x_{0}, \sigma ; \rho\right)\right)$ which agrees with $u(x, t)$ where $u$ and $v$ are both defined.

Let $E_{0}$ be the set of all sequences $\left\{a_{n}\right\}_{n=0}^{\infty}$ of real numbers. For $r>0$ let

$$
E_{r}=\left\{\left\{a_{n}\right\} \in E_{0}:\left|a_{n}\right|(2 n / e)^{n / 2}=O\left(e^{-n^{r}}\right) \text { as } n \rightarrow \infty\right\} .
$$

We call $\sup \left\{r:\left\{a_{n}\right\} \in E_{r}\right\}$ the classification number of $\left\{a_{n}\right\}$.

Explicitly, from [2, p. 222]

$$
v_{n}(x, t)=n ! \sum_{k=0}^{[n / 2]} \frac{x^{n-2 k}}{(n-2 k) !} \frac{t^{k}}{k !}, n=0,1, \cdots .
$$

In [2, Th. 5.3, p. 231] it was shown that the series $\sum_{n=0}^{\infty} a_{n} v_{n}(x, t)$ converges to a $C^{2}$-solution of the heat equation in the strip $|t|<\sigma$ where

$$
\sigma=\left(\lim \sup \left|a_{n}\right|^{2 / n}(2 n / e)\right)^{-1}
$$

and that this strip is the largest open strip of convergence of the series. One easily shows that sequences $\left\{a_{n}\right\}$ satisfying

$$
\lim \sup \left|a_{n}\right|^{2 / n}(2 n / e)=1
$$

have their classification numbers in $[0,1]$.

We will make repeated use of the following bounds which appear in [4] by S. Täcklind. Assume $u(x, t)$ is continuous on the rectangle $R=\{(x, t):|x| \leqq \mathscr{L}, 0 \leqq t \leqq T\}$, is a $C^{2}$-solution to the heat equation in the interior of $R$, and $\mu$ is an upper bound for $|u(x, t)|$ on $R$; then $u(x, t)$ is in class $C^{\infty}$ on the interior of $R$ and for $n=2,3, \cdots,|x|<$ $\mathscr{L}$, and $0<t \leqq T$

$$
\begin{aligned}
\left|\frac{\partial^{n} u}{\partial x^{n}}(x, t)\right| & \leqq \frac{\mu}{2 \sqrt{\pi}} \frac{2^{(n+3) / 2}}{t^{n / 2}} \Gamma((n+1) / 2) \\
& +\frac{\mu}{\sqrt{\pi}}\left(\frac{\pi}{2}\right)^{5 / 2} \frac{2^{3 n / 2}}{(\mathscr{L}-|x|)^{n}} \Gamma(n+1) .
\end{aligned}
$$


3. THEOREM 1. Let $\left\{X_{n}\right\}_{n=0}^{\infty}$ be a sequence of symmetric independent random variables defined on the complete probability space $(\Omega, \mathscr{F}, P)$ and satisfying

(i) there exists a number $M$ such that

$$
\int_{\Omega}\left|X_{n}(\omega)\right|^{2} d P(\omega) \leqq M \text { for } n=0,1, \cdots, \text { and }
$$

(ii) there exists $N>0$ such that

$$
N \leqq \int_{\Omega}\left|X_{n}(\omega)\right| d P(\omega), n=0,1, \cdots .
$$

Assume $\left\{a_{n}\right\}$ satisfies $\lim \sup \left|a_{n}\right|^{2 / n}(2 n / e)=1$ and has its classification number in [0,1/2). Then for almost every $\omega$ in $\Omega$ the lines $t=1$ and $t=-1$ form the natural boundary for

$$
u_{\omega}(x, t)=\sum_{n=0}^{\infty} X_{n}(\omega) a_{n} v_{n}(x, t) \text {. }
$$

Proof. Letting $\Omega^{\prime}=\left\{\omega \varepsilon \Omega: \sum_{n=0}^{\infty} X_{n}(\omega) a_{n} v_{n}(x, t)\right.$ converges in the strip $|t|<1\}$, we will first show $P\left(\Omega^{\prime}\right)=1$. Clearly

$$
\left[\lim \sup \left|X_{n}\right|^{2 / n} \leqq 1\right] \supseteq \bigcup_{k=1}^{\infty} \bigcap_{n=k}^{\infty}\left[\left|X_{n}\right| \leqq n M^{1 / 2}\right]
$$

and by the Borel-Cantelli Lemma the last set has probability 1 since $P\left[\left|X_{n}\right|>n M^{1 / 2}\right] \leqq 1 / n^{2}$ from (i). Hence

$$
P\left\{\omega: \lim \sup \left|X_{n}(\omega) a_{n}\right|^{2 / n}(2 n / e) \leqq 1\right\}=1
$$

which by (2.2) shows $P\left(\Omega^{\prime}\right)=1$.

The following fact is essentially a merger of Lemma 1 from [3] and a special case of Lemma 2 from [3]. There exist numbers $A$ in $(0,1)$ and $B>0$ with the following property: for $E \in \mathscr{F}$ with $P(E)>\Lambda$ there is a positive integer $n_{0}$ such that for $n \geqq n_{0}$, every sequence $\left\{c_{j}\right\}_{j=0}^{\infty}$ of real numbers, and $k \geqq 1$ we have

$$
\sum_{j=n}^{n+k} c_{j}^{2} \leqq B \int_{E}\left\{\sum_{j=n}^{n+k} c_{j} X_{j}(\omega)\right\}^{2} d P(\omega) \text {. }
$$

We will show that for almost every $\omega$ the line $t=-1$ is part of the natural boundary for $u_{\omega}$ and will use this in the proof for the line $t=1$.

Assume it is false that for a.e. $\omega$ in $\Omega$ the line $t=-1$ is part of the natural boundary for $u_{\omega}$. The first part of the argument we give in order to obtain a contradiction is analogous to parts of the proof of Theorem 1 in [3] by V. L. Shapiro. We will employ (2.3), (3.1), and an asymptotic estimate for heat polynomials from [2] in 
order to obtain conditions on the sequence $\left\{a_{n}\right\}$ which contradict the fact that the classification number of $\left\{a_{n}\right\}$ is in $[0,1 / 2)$.

Let $E=\left\{\omega \in \Omega^{\prime}: t=-1\right.$ is not part of the natural boundary for $\left.u_{\omega}\right\}$. Then either (i) $E \notin \mathscr{F}$, or (ii) $E \in \mathscr{F}$ and $P(E)>0$. Using the fact that the real line is separable and the countable additivity of the probability $P$, it follows that there exist a real number $x_{0}$ and $\rho_{0}>0$ such that $E_{1}=\left\{\omega \in E\right.$ : there is a $C^{2}$-solution to the heat equation in $S\left(x_{0},-1 ; \rho_{0}\right)$ which agrees with $u_{\omega}$ where they are both defined $\}$ satisfies either (i) $E_{1} \notin \mathscr{F}$, or (ii) $E_{1} \in \mathscr{F}$ and $P\left(E_{1}\right)>0$. For $i=1,2, \cdots$ define

$$
\begin{gathered}
E_{2, i}=\left\{\omega \in \Omega^{\prime}:\left|\frac{\partial^{m} u_{\omega}}{\partial x^{m}}(x, t)\right| \leqq i^{m} m^{m} \text { for }(x, t) \text { in } S\left(x_{0},-1 ; \frac{\rho_{0}}{2}\right),\right. \\
|t|<1 \text {, and } m=i, i+1, \cdots\}
\end{gathered}
$$

and let $E_{2}=\bigcup_{i=1}^{\infty} E_{2, i} \cdot \quad E_{2}$ is in the tail $\sigma$-field generated by the independent $X_{n}{ }^{\prime}$ s. From (2.3) it follows that $E_{1} \subseteq E_{2}$. By Kolmolgorov's zero-one law $P\left(E_{2}\right)=1$. Let $A$ and $B$ be as in (3.1). Take $i_{0}$ sufficiently large that $P\left(E_{2, i_{0}}\right)>\Lambda$ and let $n_{0}$ correspond to $E_{2, i_{0}}$ as in (3.1). Now let $m \geqq \max \left\{n_{0}, i_{0}\right\}$ and let $(x, t)$ be in $S\left(x_{0},-1 ; \rho_{0} / 2\right)$ with $|t|<1$. Then by (3.1) for $k=1,2, \cdots$

$$
\begin{aligned}
& \sum_{n=m}^{m+k}\left[\frac{n !}{(n-m) !} a_{n} v_{n-m}(x, t)\right]^{2} \\
& \quad \leqq B \int_{E_{2, i_{0}}}\left[\sum_{n=m}^{m+k} \frac{n !}{(n-m) !} a_{n} v_{n-m}(x, t) X_{n}(\omega)\right]^{2} d P(\omega) .
\end{aligned}
$$

Making use of the independence and symmetry of the random variables and of condition (i) we see that the integrand of the last integral is Cauchy in the variable $k$ in $L^{1}(\Omega)$ and thus in $L^{1}\left(E_{2, i_{0}}\right)$. Hence

$$
\begin{aligned}
& \sum_{n=m}^{\infty}\left[\frac{n !}{(n-m) !} a_{n} v_{n-m}(x, t)\right]^{2} \\
& \quad \leqq B \int_{E_{2, i_{0}}}\left[\sum_{n=m}^{\infty} \frac{n !}{(n-m) !} a_{n} v_{n-m}(x, t) X_{n}(\omega)\right]^{2} d P(\omega) \\
& =B \int_{E_{2, i_{0}}}\left|\frac{\partial^{m} u_{\omega}}{\partial x^{m}}(x, t)\right|^{2} d P(\omega) \leqq B i_{0}^{2 m} m^{2 m}
\end{aligned}
$$

with the last inequality following from the definition of $E_{2, i_{0}}$. We conclude that for every $m \geqq \max \left\{n_{0}, i_{0}\right\}$, every $n \geqq m$, and every $(x, t)$ in $S\left(x_{0},-1 ; \rho_{0} / 2\right)$ with $|t|<1$; we have

$$
\frac{n !}{(n-m) !}\left|a_{n}\right|\left|v_{n-m}(x, t)\right| \leqq B^{1 / 2} i_{0}^{m} m^{m}
$$


It follows from Theorem 3.1 of [2] that there exist numbers $A$ and $l_{0}$ such that for $n \geqq l_{0}$

$$
\sup _{\left|x-x_{0}\right|<\rho_{0} / 2}\left|v_{n}(x,-1)\right| \geqq A[2 n / e]^{n / 2} .
$$

Thus from (3.2) we have for $n>m+l_{0}>m \geqq \max \left\{n_{0}, i_{0}\right\}$

$$
\left|a_{n}\right| \frac{n !}{(n-m) !} A[2(n-m) / e]^{(n-m) / 2} \leqq B^{1 / 2} i_{0}^{m} m^{m} .
$$

Employing Stirling's theorem we see there is a number $C$ such that for $n>m+l_{0}>m \geqq \max \left\{n_{0}, i_{0}\right\}$

$$
\left|a_{n}\right|(2 n / e)^{n / 2} \leqq\left[\frac{C m}{\sqrt{n-m}}\right]^{m} \cdot((n-m) / n)^{(n+1) / 2} .
$$

Let $r$ be a number which is strictly greater than the classification number of $\left\{a_{n}\right\}$ and strictly less than $1 / 2$. Let $m$ be related to $n$ by $m=\left[4 n^{r}\right]+1$ where the brackets denote the greatest integer function. Then from (3.3), for sufficiently large $n$,

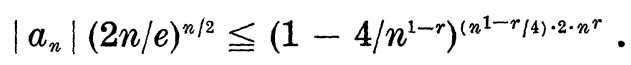

For large enough $n,\left(1-4 / n^{1-r}\right)^{\left(n^{1-r / 4) \cdot 2}\right.} \leqq 1 / e$ and thus from (3.4) we have for such $n,\left|a_{n}\right|(2 n / e)^{n / 2} \leqq 1 / e^{n^{r}}$. Hence $\left\{a_{n}\right\} \in E_{r}$ which contrandicts the fact that $r$ is strictly greater than the classification number of $\left\{a_{n}\right\}$ and concludes the proof for the line $t=-1$.

For the last part of the proof we find it convenient to introduce the probability space $\left(R^{\omega}, \mathscr{X}^{\prime}, \mu^{\prime}\right)$ which we now describe.

$$
R^{\omega}=\prod_{n=0}^{\infty} R_{n}
$$

where each $R_{n}$ is the set of real numbers. Let $\mathscr{A}_{0}$ be the field of all subsets of $R^{\omega}$ of the form $B \times\left(\prod_{n=n_{0}+1}^{\infty} R_{n}\right)$ where $n_{0}$ is a positive integer and $B$ is a Borel set in $\prod_{n=0}^{n_{0}} R_{n}$. Let $\mathscr{A}$ be the $\sigma$-field generated by $\mathscr{A}_{0}$. Let $\mu$ be the probability on $\left(R^{\omega}, \mathscr{A}\right)$ which is induced by the $X_{n}^{\prime}$ 's. Then $\left(R^{\omega}, \mathscr{A}^{\prime}, \mu^{\prime}\right)$ is the completion of $\left(R^{\omega}, \mathscr{A}\right.$, $\mu)$.

Let $\left\{\eta_{i}\right\}_{i=0}^{\infty}$ be a sequence of $\pm 1^{\prime}$ s. Define $T: R^{\omega} \rightarrow R^{\omega}$ by

$$
T\left(\left(\xi_{0}, \xi_{1}, \cdots\right)\right)=\left(\eta_{0} \xi_{0}, \eta_{1} \xi_{1}, \cdots\right) .
$$

Notice that

$$
\begin{aligned}
\mu\left(\prod_{n=0}^{n_{0}}\left(a_{n}, b_{n}\right] \times \prod_{n=n_{0}+1}^{\infty} R_{n}\right) & =\prod_{n=0}^{n_{0}} P\left[X_{n} \in\left(a_{n}, b_{n}\right]\right] \\
= & \prod_{n=0}^{n_{0}} P\left[X_{n} \in \eta_{n}\left(a_{n}, b_{n}\right]\right]=\mu\left(T\left(\prod_{n=0}^{n_{0}}\left(a_{n}, b_{n}\right] \times \prod_{n=n_{0}+1}^{\infty} R_{n}\right)\right)
\end{aligned}
$$


where we have used both the independence and symmetry of the $X_{n}$ 's. From this it follows that for $A \in \mathscr{C}^{\prime}, \mu^{\prime}(A)=\mu^{\prime}(T(A))$. We will make use of this fact twice in the remainder of this proof.

To finish the proof it suffices to show that for a.e. $p \in R^{\omega}$ the line $t=1$ is part of the natural boundary for

$$
u_{p}(x, t)=\sum_{n=0}^{\infty} \pi_{n}(p) a_{n} v_{n}(x, t)
$$

where the $\pi_{n}$ 's are the projection random variables. Suppose this is false. From the first paragraph of the present proof we know $R^{w \prime}=$ $\left\{p \in R^{\omega}: \sum_{n=0}^{\infty} \pi_{n}(p) a_{n} v_{n}(x, t)\right.$ converges in $\left.|t|<1\right\}$ has $\mu^{\prime}$-measure 1 . Now let $F=\left\{p \in R^{(\omega \prime}: t=1\right.$ is not part of the natural boundary for $u_{p}$ \}. Then either (i) $F \in \mathscr{C}^{\prime}$, or (ii) $F \in \mathscr{C}^{\prime}$ and $\mu^{\prime}(F)>0$. It follows that there exist numbers $a, b, \rho$ with $a<b$ and $\rho>0$ such that $F_{1}=$ $\left\{p \in R^{\omega \prime}\right.$ : there is a function $v_{p}(x, t)$ which is continuous on $a \leqq x \leqq b$, $0 \leqq t \leqq 1+\rho$; is a $C^{2}$-solution to the heat equation for $a<x<b$, $0<t<1+\rho$; and agrees with $u_{p}(x, t)$ in $\left.a \leqq x \leqq b, 0 \leqq t<1\right\}$ satisfies either (i) $F_{1} \in \mathscr{C}^{\prime}$, or (ii) $F_{1} \in \mathscr{C}^{\prime}$ and $\mu^{\prime}\left(F_{1}\right)>0$. But $F_{1}=\left\{p \in R^{\omega \prime}\right.$ : $\lim _{t \uparrow 1} u_{p}(a, t)$ and $\lim _{t \uparrow 1} u_{p}(b, t)$ both exist $\} . F_{1}$ is in the tail $\sigma$-field generated by the independent $\pi_{n}$ 's. From the zero-one law, $\mu^{\prime}\left(F_{1}\right)=1$.

Either $a \neq 0$ or $b \neq 0$ and for definiteness we assume $a \neq 0$. Then $F_{2}=\left\{p \in R^{\omega \prime}: \lim _{t \uparrow 1} u_{p}(a, t)\right.$ exists $\}$ has $\mu^{\prime}\left(F_{2}\right)=1$. Let $T: R^{\omega} \rightarrow R^{w}$ be defined by $T\left(\left(\xi_{0}, \xi_{1}, \cdots\right)\right)=\left(\xi_{0},-\xi_{1}, \xi_{2},-\xi_{3}, \cdots\right)$. By our earlier comments concerning such mappings we have $\mu^{\prime}\left(F_{2} \cap T\left(F_{2}\right)\right)=1$. For $p \in R^{\omega \prime}$ and $|t|<1$ one checks that $u_{T(p)}(-a, t)=u_{p}(a, t)$. Hence for $p \in F_{2} \cap T\left(F_{2}\right), \lim _{t \uparrow 1} u_{p}(-a, t)$ and $\lim _{t \uparrow 1} u_{p}(a, t)$ both exist. Thus for $p \in F_{2} \cap T\left(F_{2}\right)$ there is a function $w_{p}(x, t)$ which is continuous in $|x| \leqq$ $a, 0 \leqq t \leqq 2$; is a $C^{2}$-solution to the heat equation in $|x|<a, 0<t<2$; and agrees with $u_{p}$ in $|x| \leqq a, 0 \leqq t<1$. For $p \in F_{2} \cap T\left(F_{2}\right)$ and $0 \leqq$ $t \leqq 2$ let $\phi_{p}(t)=w_{p}(0, t)$ and $\dot{\gamma}_{p}(t)=\left(\partial w_{p} / \partial x\right)(0, t)$. Then, employing (2.3), we see that $\dot{\phi}_{p}$ and $\psi_{p}$ are in class $C\{(2 n) !\}$ on $[0,2]$ (a function $f$ is in class $C\{(2 n) !\}$ on an interval $I$ if $f$ is in class $C^{\infty}$ on $I$ and there exist constants $\beta$ and $B$ such that for every $t$ in $I,\left|f^{(n)}(t)\right| \leqq$ $\left.\beta B^{n}(2 n) !, n=0,1, \cdots\right)$.

Now let $T^{\prime}: R^{\omega} \rightarrow R^{\omega}$ be defined by

$$
T^{\prime}\left(\left(\xi_{0}, \xi_{1}, \cdots\right)\right)=\left(\xi_{0}, \xi_{1},-\xi_{2},-\xi_{3}, \xi_{4}, \xi_{5},-\xi_{6},-\xi_{7}, \cdots\right) .
$$

Then for $p \in R^{\omega \prime}$ and $|t|<1, u_{p}(0, t)=u_{T^{\prime}(p)}(0,-t)$ and

$$
\frac{\partial u_{p}}{\partial x}(0, t)=\frac{\partial u_{T^{\prime}(p)}}{\partial x}(0,-t) \text {. }
$$

For $p$ in the almost sure set $T^{\prime}\left(F_{2} \cap T\left(F_{2}\right)\right)$ we have $T^{\prime}(p) \in F_{2} \cap T\left(F_{2}\right)$ and we define $\phi_{p}^{\prime}$ and $\psi_{p}^{\prime}$ on $[-2,0]$ by $\phi_{p}^{\prime}(t)=\phi_{T^{\prime}(t)}(-t)$ and

$$
\psi_{p}^{\prime}(t)=\psi_{T^{\prime}(p)}(-t)
$$


thereby obtaining class $C\{(2 n) !\}$ extensions of $u_{p}(0, t)$ and $\left(\partial u_{p} / \partial x\right)(0, t)$ on $[-1,0]$. Thus for $p \in T^{\prime}\left(F_{2} \cap T\left(F_{2}\right)\right)$

$$
u_{p}^{\prime}(x, t)=\sum_{n=0}^{\infty} \frac{\phi_{p}^{\prime(n)}(t) x^{2 n}}{(2 n) !}+\sum_{n=0}^{\infty} \frac{\psi_{p}^{\prime(n)}(t) x^{2 n+1}}{(2 n+1) !}
$$

provides a solution to the heat equation which is a $C^{2}$-extension of $u_{p}$ into some rectangle $|x|<r,-2<t<0$ which contradicts the first part of the proof.

4. THeOREM 2. Let $\left\{X_{n}\right\}$ be a sequence of independent random variables over a probability space $(\Omega, \mathscr{F}, P)$ which satisfies (i) and (ii) of Theorem 1. Assume $\left\{a_{n}\right\}$ satisfies $\lim \sup \left|a_{n}\right|^{2 / n}(2 n / e)=1$ and has its classification number in $(1 / 2,1]$. Then for almost every $\omega$ in $\Omega$ the following holds: $|t|<1$ is the strip of convergence of $\sum_{n=0}^{\infty} X_{n}(\omega) a_{n} v_{n}(x, t)$ which for every $\mathscr{L}>0$ can be extended as a $C^{2}$-solution of the heat equation into $\{|t|<1\} \cup\{|x|<\mathscr{L}\}$.

Proof. We will first show for almost every $\omega$ in $\Omega$ that $|t|<1$ is the strip of convergence of $\sum_{n=0}^{\infty} X_{n}(\omega) a_{n} v_{n}(x, t)$. By (2.2) we must show that almost surely $\lim \sup \left|X_{n}(\omega) a_{n}\right|^{2 / n}(2 n / e)=1$. The argument given in the first part of the proof of Theorem 1 shows that almost surely the last limit superior does not exceed 1 . Let $\left\{n_{j}\right\}$ be a strictly increasing sequence of positive integers such that

$$
\lim _{j \rightarrow \infty}\left|a_{n_{j}}\right|^{2 / n_{j}}\left(2 n_{j} / e\right)=1 \text {. }
$$

Then $\lim \sup \left|X_{n}(\omega) a_{n}\right|^{2 / n}(2 n / e) \geqq \lim \sup _{j \rightarrow \infty}\left|X_{n_{j}}(\omega) a_{n_{j}}\right|^{2 / n_{j}}\left(2 n_{j} / e\right) \geqq \lim$ $\sup _{j \rightarrow \infty}\left|X_{n_{j}}(\omega)\right|^{2 / n_{j}}$ which by the zero-one law is equal to some number $c$ almost surely. Suppose $c<1$. Then $X_{n_{j}} \rightarrow 0$ almost surely. By (ii) for $A>0$ and $j=0,1, \ldots$

$$
N \leqq \int_{\left[\left|X_{n j}\right| \leqq A\right]}\left|X_{n_{j}}(\omega)\right| d P(\omega)+A^{-1} \int_{\left[\left|X_{n j}\right|>A\right]}\left|X_{n_{j}}(\omega)\right|^{2} d P(\omega) .
$$

By the Lebesgue dominated convergence theorem the next to the last integral tends to 0 as $j$ tends to $\infty$. From (i) the last term is uniformly bounded by $A^{-1} M$. Thus for every $A>0, N \leqq A^{-1} M$ which is a contradiction. We conclude that $c \geqq 1$. Thus almost surely

$$
\lim \sup \left|X_{n}(\omega) a_{n}\right|^{2 / n}(2 n / e) \geqq 1
$$

which concludes the proof that almost surely this limit superior is 1 .

In order to establish Theorem 2 for the line $t=1$ we first construct a function which is $C^{\infty}$ on the closed strip $|t| \leqq 1$ and has a heat polynomial expansion in $|t|<1$. Let $r$ be a number which is strictly greater than $1 / 2$ and strictly less than the classification num- 
ber of $\left\{a_{n}\right\}$. For $n=0,1, \cdots$ define $\alpha_{n}=(2 n) e^{-n^{r}}$. Define $f$ on $[-1,1]$ by $f(t)=\sum_{k=0}^{\infty} \alpha_{k} t^{k}$. We will show this definition makes sense and obtain some bounds on the derivatives of $f$.

Let $n$ be a nonnegative integer. Differentiating $\sum_{k=0}^{\infty} \alpha_{k} t^{k}$ term by term $n$ times yields $\sum_{k=n}^{\infty} k ! /(k-n) ! a_{k} t^{k-n}$. For $|t| \leqq 1$ the $k^{\text {th }}$ term of this series is dominated by $2 k^{n+1} e^{-k^{r}}$. One checks that

$$
g_{n}(x)=x^{n+1} e^{-x^{r}}
$$

is increasing on $\left(0,(n+1 / r)^{1 / r}\right)$ and decreasing on $\left((n+1 / r)^{1 / r}, \infty\right)$. Hence

$$
\sum_{k=n}^{\infty} k^{n+1} e^{-k r} \leqq \int_{n}^{\infty} g_{n}(x) d x+g_{n}\left(\left(\frac{n+1}{r}\right)^{1 / r}\right) \leqq 3 \Gamma((n+2) / r) / r .
$$

We conclude that $f$ is a $C^{\infty}$-function with $\left|f^{(n)}(t)\right| \leqq 6 \Gamma((n+2 / r) / r$ for $n=0,1, \cdots$ and $|t| \leqq 1$.

Now define

$$
u(x, t)=\sum_{n=0}^{\infty} \frac{f^{(n)}(t) x^{2 n}}{(2 n) !}+\sum_{n=0}^{\infty} \frac{f^{(n+1)}(t) x^{2 n+1}}{(2 n+1) !} .
$$

Because of the bounds obtained in the preceding paragraph it can be shown that the series of (4.1) can be differentiated term by term and that $u(x, t)$ is a $C^{\infty}$-solution to the heat equation in the closed strip $|t| \leqq 1$. Since both $u(0, t)$ and $\partial u / \partial x(0, t)$, as functions of $t$ on $(-1,1)$, are given by their Maclaurin expansions, $u$ has a heat polynomial expansion in $|t|<1$ (see [5]). Thus

$$
\begin{aligned}
u(x, t) & =\sum_{n=0}^{\infty} b_{n} v_{n}(x, t), \\
b_{2 n} & =f^{(n)}(0) /(2 n) !, \\
b_{2 n+1} & =f^{(n+1)}(0) /(2 n+1) ! .
\end{aligned}
$$

According to the first paragraph of the proof of Theorem 1, $\bigcup_{k=1}^{\infty} \bigcap_{n=k}^{\infty}\left[\left|X_{n}\right| \leqq n M^{1 / 2}\right]$ has probability 1 . Let $\omega$ be in this almost sure set. Let $k_{0}$ be a positive integer such that for $n \geqq k_{0},\left|X_{n}(\omega)\right| \leqq$ $n M^{1 / 2}$. Since $r$ is less than the classification number of $\left\{a_{n}\right\}$, there is a number $K$ such that $\left|a_{n}\right|(2 n / e)^{n / 2} \leqq K e^{-n^{r}}, n=1,2, \cdots$. Using Stirling's theorem we have for $2 n \geqq k_{0}$

$$
b_{2 n}(4 n / e)^{n} \geqq\left|X_{2 n}(\omega) a_{2 n}\right|(4 n / e)^{n}(1 / 2)^{3 / 2} / K M^{1 / 2} .
$$

Similarly for $2 n+1 \geqq k_{0}$

$$
b_{2 n+1}(2(2 n+1) / e)^{(2 n+1) / 2} \geqq\left|X_{2 n+1}(\omega) a_{2 n+1}\right|(2(n+1) / e)^{(2 n+1) / 2} e^{-1 / 2} / K M^{1 / 2} .
$$

Letting $K^{\prime}=K(M e)^{1 / 2}$ we have 


$$
\left|X_{n}(\omega) a_{n}\right| \leqq K^{\prime} b_{n} \text { for } n \geqq k_{0} \text {. }
$$

Let $\mathscr{L}>0$. Then for $0<t<1$ we have

$$
\begin{aligned}
& \left|\frac{\partial}{\partial t} \sum_{n=k_{0}}^{\infty} X_{n}(\omega) a_{n} v_{n}( \pm \mathscr{L}, t)\right|=K^{\prime} \sum_{n=k_{0}}^{\infty} b_{n} n(n-1)\left|v_{n-2}( \pm \mathscr{L}, t)\right| \\
& \leqq K^{\prime} \sum_{n=k_{0}}^{\infty} b_{n} n(n-1) v_{n-2}(\mathscr{L}, t) \leqq K^{\prime} \frac{\partial u}{\partial t}(\mathscr{L}, 1)<\infty .
\end{aligned}
$$

Thus $\lim _{t \uparrow 1} \sum_{n=k_{0}}^{\infty} X_{n}(\omega) a_{n} v_{n}( \pm \mathscr{L}, t)$ both exist as is easily seen from the mean value theorem and the Cauchy criterion. Hence we can obtain an extension of $\sum_{n=0}^{\infty} X_{n}(\omega) a_{n} v_{n}(x, t)$ into

$$
\{(x, t):|t|<1\} \bigcup\{(x, t):|x|<\mathscr{L}, 0<t\}
$$

which is a $C^{2}$-solution of the heat equation. (Notice at this point that we can also obtain an extension which is a bounded $C^{2}$-solution in $\{(x, t):|x|<\mathscr{L}, 0 \leqq t\}$.) Since $\omega$ was from the almost sure set

$$
\bigcup_{k=1}^{\infty} \bigcap_{n=k}^{\infty}\left[\left|X_{n}\right| \leqq n M^{1 / 2}\right] \text {, }
$$

this establishes the result for the line $t=1$.

We now turn to the line $t=-1$. Define $\left\{Y_{n}\right\}_{n=0}^{\infty}$ on $\Omega$ by $Y_{2 n}=$ $(-1)^{n} X_{2 n}$ and $Y_{2 n+1}=(-1)^{n} X_{2 n+1}$. Then, applying the first part of the proof, there is a set $F$ in $\mathscr{F}$ with $P(F)=1$ such that for $\omega$ in $F$ and $\mathscr{L}>0$ the solution $v_{\omega}(x, t)=\sum_{n=0}^{\infty} Y_{n}(\omega) a_{n} v_{n}(x, t)$ can be extended into $\{|t|<1\} \bigcup\{|x|<\mathscr{L}$ and $0<t\}$ so as to be a bounded $C^{2}$-solution of the heat equation in $\{(x, t):|x|<\mathscr{L}$ and $0<t\}$. One easily checks that for $\omega$ in $F$,

$$
\sum_{n=0}^{\infty} X_{n}(\omega) a_{n} v_{n}(0, t)=\sum_{n=0}^{\infty} Y_{n}(\omega) a_{n} v_{n}(0,-t)
$$

and $\sum_{n=1}^{\infty} X_{n}(\omega) a_{n} n v_{n-1}(0, t)=\sum_{n=1}^{\infty} Y_{n}(\omega) a_{n} n v_{n-1}(0,-t)$. Using these facts and (2.3) we see that for $\omega$ in $F$ and $\mathscr{L}>0$ the functions $\dot{\phi}(t)=\sum_{n=0}^{\infty} X_{n}(\omega) a_{n} v_{n}(0, t)$ and $\psi(t)=\sum_{n=1}^{\infty} X_{n}(\omega) a_{n} n v_{n-1}(0, t)$ on $(-1,1)$ possess sufficiently well behaved extensions $\phi^{\prime}$ and $\psi^{\prime}$ to $(-\infty, 1)$ that

$$
\sum_{n=0}^{\infty} \frac{\phi^{\prime(n)}(t) x^{2 n}}{(2 n) !}+\sum_{n=0}^{\infty} \frac{\psi^{\prime(n)}(t) x^{2 n+1}}{(2 n+1) !}
$$

is an extension of $\sum_{n=0}^{\infty} X_{n}(\omega) a_{n} v_{n}(x, t)$ in $|t|<1$ to

$$
\{(x, t):|t|<1\} \bigcup\{(x, t):|x|<\mathscr{L} \text { and }-\infty<t<1\} \text {. }
$$

5. Examples. The first example will show that our two theorems are best possible with respect to the allowable values of the classification number. 
EXAMPLE 1. We will take $[0,1]$ with Lebesgue measure as the probability space and the sequence of Rademacher functions, $\left\{\hat{\phi}_{n}\right\}_{n=0}^{\infty}$, for the random variables.

For $k=0,1, \cdots$ define $\alpha_{k}=e^{-\sqrt{k}}$. Then, as in the proof of Theorem 2, defining $f$ on $[-1,1]$ by $f(t)=\sum_{k=0}^{\infty} \alpha_{k} t^{k}$ yields a $C^{\infty}$-function whose $n^{\text {th }}$ derivative on $[-1,1]$ is bounded in absolute value by $6 \Gamma(2(2 n+1))$. In the strip $|t|<1$ define $u(x, t)=\sum_{n=0}^{\infty}\left(f^{(n)}(t) x^{2 n}\right) /(2 n) !$. To see that this definition makes sense and that term by term partial differentiation is permitted, we note that for every closed interval $I \subseteq(-1,1), f$ is in class $C\{n !\}$ on $I$. Because of the bounds on the derivatives of $f$ we see from the defining series for $u$ that $u$ may be extended as a $C^{\infty}$-solution of the heat equation to

$$
\{|t|<1\} \bigcup\{(x, 1):|x|<1\} \text {. }
$$

Since $u(0, t)$ and $\partial u / \partial x(0, t)$ are both given by their Maclaurin expansions in $|t|<1, u$ possesses a heat polynomial expansion in the strip $|t|<1$ (see [5]). Thus for $|t|<1, u(x, t)=\sum_{n=0}^{\infty} a_{n} v_{n}(x, t) ; a_{2 n}=$ $\left(e^{-\sqrt{n}} n !\right) /(2 n) !, a_{2 n+1}=0$. One checks that $\lim \sup \left|a_{n}\right|^{2 / n}(2 n / e)=1$. Also it is easily seen that limit $\left|a_{2 n}\right|(4 n / e)^{n} e^{\sqrt{2 n}}=\infty$ which implies $\left\{a_{n}\right\} \notin E_{1 / 2}$ and thus the classification number of $\left\{a_{n}\right\}$ is in $[0,1 / 2]$. As in the proof of Theorem $2, \lim _{t \uparrow 1} u_{\omega}( \pm 1 / 2, t)$ both exist for every $\omega$ in $[0,1]$. Thus for every $\omega \in[0,1]$ the line $t=1$ is not part of the natural boundary for $u_{\omega}(x, t)$. Using Theorem 1 , we conclude that the classification number of $\left\{a_{n}\right\}$ is $1 / 2$ and that in Theorem 1 we cannot replace $[0,1 / 2)$ by $[0,1 / 2]$ as the allowable range for the classification number.

We will next show that the conclusion of Theorem 2 does not hold for $\sum_{n=0}^{\infty} \phi_{n}(\omega) a_{n} v_{n}(x, t)$. Assume there is a set $A$ in $[0,1]$ with $m(A)=1$ such that for each $\omega$ in $A$ no interval of the line $t=1$ is part of the natural boundary for $u_{\omega}(x, t)$. Thus for $\omega$ in $A, g_{\omega}(x)=$ $\lim _{t \uparrow 1} u_{\omega}(x, t)$ is well defined and is the restriction of an entire function to the real axis (this last assertion can be seen by employing (2.3)). Thus for $\omega$ in $A$, $\lim \sup \left(\left|g_{\omega}^{(n)}(0)\right| / n !\right)^{1 / n}=0$. For $\omega$ in $A$, $\left|g_{\omega}^{(2 n+1)}(0)\right|=0$ and $\left|g_{\omega}^{(2 n)}(0)\right|=\left|\sum_{k=2 n}^{\infty} \phi_{k}(\omega) a_{k}(k ! /(k-2 n) !) v_{k-2 n}(0,1)\right|=$ $\left|\sum_{k=n}^{\infty} \phi_{2 k}(\omega)(k ! /(k-n) !) e^{-\sqrt{k}}\right|$. Thus for $\omega$ in $A$,

$$
\lim \sup \left[\frac{\left|\sum_{k=n}^{\infty} \phi_{2 k}(\omega) \frac{k !}{(k-n) !} e^{-\sqrt{k}}\right|}{(2 n) !}\right]^{1 / n}=0 .
$$

Let $\delta>0$. For $m=0,1, \cdots$ let

$$
\begin{aligned}
F_{m} & =\left\{\omega \in A:\left(\left|\sum_{k=n}^{\infty} \phi_{2 k}(\omega) \frac{k !}{(k-n) !} e^{-\sqrt{k}}\right| /(2 n) !\right)^{1 / n}\right. \\
& \leqq \delta \text { for } n=m, m+1, \cdots\}
\end{aligned}
$$


and note $F_{m} \uparrow A$. Let $A$ and $B$ be two numbers associated with the sequence $\left\{\dot{\phi}_{2 n}\right\}_{n=0}^{\infty}$ as in (3.1). Let $m_{0}$ be sufficiently large that $m\left(F_{m_{0}}\right)>$ 1. Let $n_{0}$ be an integer larger than $m_{0}$ with $n_{0}$ corresponding to $F_{m_{0}}$ as in (3.1). Thus for $n \geqq n_{0}$ and $k \geqq 1$

$$
\sum_{j=n}^{n+l k}\left[\frac{j !}{(j-n) !} e^{-\sqrt{j}}\right]^{2} \leqq B \int_{F m_{0}}\left(\sum_{j=n}^{n+k} \dot{\phi}_{2 j}(\omega) \frac{j !}{(j-n) !} e^{-\sqrt{j}}\right)^{2} d m(\omega)
$$

As in the proof of Theorem 1, letting $k$ tend to $\infty$ yields (5.1) with $n+k$ replaced by $\infty$. Using the definition of $F_{m_{0}}$, we have

$$
\sum_{j=n}^{\infty}\left[\frac{j !}{(j-n) !} e^{-\sqrt{j}}\right]^{2} \leqq B\left((2 n) ! \delta^{n}\right)^{2},
$$

for $n \geqq n_{0}$. From this we conclude that

$$
\lim \sup \left[\frac{\left[\sum_{k=n}^{\infty}\left(\frac{k !}{(k-n) !} e^{-\sqrt{k}}\right)^{2}\right]^{1 / 2}}{(2 n) !}\right]^{1 / n}=0 .
$$
have

On the other hand, letting $L$ denote this last limit superior, we $L \geqq \lim \sup$

$$
\left[\frac{\left[\sum_{k=n}^{\infty}(k-n)^{2 n} \exp (-2 \sqrt{k-n}) \exp (-(2 \sqrt{k}-2 \sqrt{k-n}))\right]^{1 / 2}}{(2 n) !}\right]^{1 / n} .
$$

But $\exp (-(2 \sqrt{k}-2 \sqrt{k-n})) \geqq e^{-2 \sqrt{ } n}$ for $k \geqq n$ and $\lim \left(e^{-\sqrt{n}}\right)^{1 / n}=$ 1. Hence $L \geqq \limsup \left(\left(\sum_{k=0}^{\infty} k^{2 n} e^{-2 \sqrt{k}}\right)^{1 / 2} /(2 n) !\right)^{1 / n}$. Define $h_{n}$ on $(0, \infty)$ by $h_{n}(x)=x^{2 n} e^{-2 \sqrt{x}}$. One checks that $h_{n}$ is increasing on $\left(0,(2 n)^{2}\right)$ and decreasing on $\left((2 n)^{2}, \infty\right)$. Thus $\sum_{k=0}^{\infty} k^{2 n} e^{-2 \sqrt{k}} \geqq \int_{0}^{\infty} h_{n}(x) d x-h_{n}\left((2 n)^{2}\right)=$ $\left(\Gamma(4 n+2)-2(4 n)^{4 n} e^{-4 n}\right) /\left(2 \cdot 4^{2 n}\right)$. Thus

$$
L \geqq \frac{1}{4} \lim \sup \left[\left(\frac{\Gamma(4 n+2)}{(4 n) !}-\frac{2(4 n)^{4 n} e^{-4 n}}{(4 n) !}\right)\left((4 n) ! /((2 n) !)^{2}\right)\right]^{1 / 2 n}>0 .
$$

This is a contradiction. Hence in Theorem 2 we cannot replace $(1 / 2,1]$ by $[1 / 2,1]$ as the allowable range for the classification number.

The next example shows that in Theorem 1 we cannot omit the symmetry of the random variables.

ExAMPLE 2. Let $k(x, t)=e^{-x^{2} / 4 t} /(4 \pi t)^{1 / 2}$ for $t>0$ and define

$$
u(x, t)=k(x, t+1)
$$

in the strip $|t|<1$. Then [2, Th. 4.2, p. 227] 


$$
u(x, t)=(4 \pi)^{-1 / 2} \sum_{n=0}^{\infty} \frac{(-1)^{n}}{n ! 4^{n}} v_{2 n}(x, t) .
$$

Let $\left\{a_{n}\right\}_{n=0}^{\infty}$ be defined by $a_{2 n}=(-1)^{n} / n ! 4^{n}$ and $a_{2 n+1}=0$. One easily checks that $\lim \sup \left|a_{n}\right|^{2 / n}(2 n / e)=1$ and that the classification number of $\left\{a_{n}\right\}=0$. Let $X_{n}=1, n=0,1, \cdots$ on some complete probability space. Then for every $\omega, u_{\omega}$ can be continued above the line $t=1$.

\section{BIBLIOGRAPHY}

1. J. P. Kahane, Sèries de Fourier aleatoires, les presses de l'Universite de Montreal, Montreal, 1966.

2. P. C. Rosenbloom and D. V. Widder, Expansions in terms of heat polynomials and associated functions, Trans. Amer. Math. Soc. 92 (1959), 220-266.

3. V. L. Shapiro, "Spherical caps and random valued harmonic functions," New directions in orthogonal expansions and their continuous analogues, Southern Illinois University Press, 1968.

4. S. Täcklind, Sur les classes quasianalytiques des solutions equations aux derivees partielles du type parabolique, Nova Acta Soc. Sci. Upsall Ser. IV, 10 (1936), 1-56.

5. D. V. Widder, Analytic solutions of the heat equation, Duke Math. J. 29 (1962), 497-504.

6. A. Zygmund, Trigonometric series, Vol. I, Cambridge Univ. Press, Cambridge, 1959 .

Received July 23, 1968. This paper is based on part of the author's doctoral dissertation at the University of California at Riverside which was written under the direction of Victor L. Shapiro. Research sponsored by the Air Force Office of Scientific Research, Office of Aerospace Research, United States Air Force, under AFOSR Grant No. AF-AFOSR 694-66.

UNiversity of CALifornia at Riverside

UNIVERSITY OF KENTUCKY 


\section{PACIFIC JOURNAL OF MATHEMATICS}

\section{EDITORS}

H. ROYDEN

Stanford University

Stanford, California

Richard Pierce

University of Washington

Seattle, Washington 98105
J. DUGUNDJI

Department of Mathematics

University of Southern California

Los Angeles, California 90007

BASIL GORDON

University of California

Los Angeles, California 90024

\section{ASSOCIATE EDITORS}
E. F. BECKENBACH
B. H. NEUMANN
F. WOLF
K. YosHIDA

\section{SUPPORTING INSTITUTIONS}

UNIVERSITY OF BRITISH COLUMBIA

CALIFORNIA INSTITUTE OF TECHNOLOGY

UNIVERSITY OF CALIFORNIA

MONTANA STATE UNIVERSITY

UNIVERSITY OF NEVADA

NEW MEXICO STATE UNIVERSITY

OREGON STATE UNIVERSITY

UNIVERSITY OF OREGON

OSAKA UNIVERSITY

UNIVERSITY OF SOUTHERN CALIFORNIA
STANFORD UNIVERSITY

UNIVERSITY OF TOKYO

UNIVERSITY OF UTAH

WASHINGTON STATE UNIVERSITY

UNIVERSITY OF WASHINGTON

*

AMERICAN MATHEMATICAL SOCIETY CHEVRON RESEARCH CORPORATION TRW SYSTEMS

NAVAL WEAPONS CENTER 


\section{Pacific Journal of Mathematics}

\section{Vol. 31, No. $1 \quad$ November, 1969}

James Burton Ax, Injective endomorphisms of varieties and schemes........

Richard Hindman Bouldin, A generalization of the Weinstein-Aronszajn

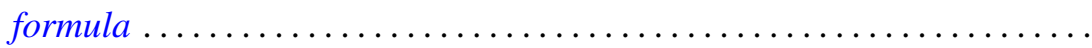

John Martin Chadam, The asymptotic behavior of the Klein-Gordon equation with external potential. II ...............................

Rina Hadass, On the zeros of the solutions of the differential equation

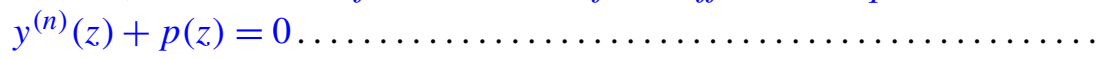

John Sollion Hsia, Integral equivalence of vectors over local modular lattices. II .............................................

Robert Hughes, Boundary behavior of random valued heat polynomial

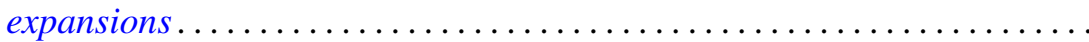

Surender Kumar Jain, Saad H. Mohamed and Surjeet Singh, Rings in which every right ideal is quasi-injective .........................

T. Kawata, On the inversion formula for the characteristic function .........

Erwin Kleinfeld, On right alternative rings without proper right ideals......

Robert Leroy Kruse and David Thomas Price, On the subring structure of

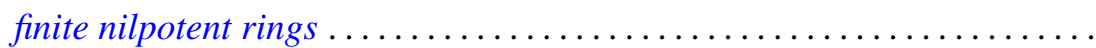

Marvin David Marcus and Stephen J. Pierce, Symmetric positive definite multilinear functionals with a given automorphism. .

William Schumacher Massey, Pontryagin squares in the Thom space of a bundle...

William Schumacher Massey, Proof of a conjecture of Whitney ...

John William Neuberger, Existence of a spectrum for nonlinear transformations

Stephen E. Newman, Measure algebras on idempotent semigroups ...

$\mathrm{K}$. Chandrasekhara Rao, Matrix transformations of some sequence spaces

Robert Bruce Schneider, Some theorems in Fourier analysis on symmetric

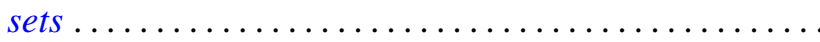

Ulrich F. K. Schoenwaelder, Centralizers of abelian, normal subgroups of hypercyclic groups...

Jerrold Norman Siegel, $G$-spaces, $H$-spaces and $W$-spaces

Robert Irving Soare, Cohesive sets and recursively enumerable Dedekind cuts...

Kwok-Wai Tam, Isometries of certain function spaces .... . .

Awadhesh Kumar Tiwary, Injective hulls of semi-simple modules over regular rings ....

Eldon Jon Vought, Concerning continua not separated by any nonaposyndetic subcontinuum .................... 